\title{
Routability Crossing Distribution and Floating Pin Assignment for $T$-type Junction Region
}

\author{
JIN-TAI YAN \\ Computer Systems Research Center, National Chiao Tung University, Hsinchu, Taiwan, R.O.C.
}

(Received 15 July 1997; In final form 8 July 1998)

\begin{abstract}
Based on non-crossing relations, vertical constraint relations and net-geometry relations in one $T$-type junction region, two kinds of routability crossing distributions are proposed to improve routing performance in one $T$-type junction region. For routability crossing distribution in one $T$-type junction region, a routability-ordering graph is built to decide a net ordering in $O\left(n^{2}\right)$ time. For routability quota crossing distribution in one $T$-type junction region, if the number of net crossings in base channel is more than a given quota, this net ordering in routability crossing distribution will be further adjusted to satisfy the quota requirement by using a net interchange operation in $O(n)$ time. For floating pin assignment in one $T$-type junction region, global nets are assigned on the boundary between top channel and base channel by interleaving vacant pins in $O(n)$ time according to a net ordering in routability crossing distribution.
\end{abstract}

Keywords: Physical design, placement, global routing, detailed routing, routing ordering, crossing distribution

\section{INTRODUCTION}

In a building block layout [1-2], the placement/ routing process is divided into the following phases: placement, global-routing, region definition and ordering assignment (RDAOA), and detailed-routing. In general, according to the constraints of layout area or routing performance, all the building blocks are placed on fixed positions, and routing space between any pair of adjacent building blocks is assigned to route all the nets in the placement phase. After the placement phase, all the routing nets are globally assigned within the routing space in the global-routing phase. Furthermore, the routing space is partitioned and defined into straight channels, switchboxes, or $L$-shaped channels, and these defined regions are assigned a safe routing ordering in the RDAOA phase. Finally, all the straight channels, switchboxes, and $L$-shaped channels are routed by a straight channel router, a switchbox router and an $L$-shaped channel router in the detailed-routing phase, respectively.

Consider a given building block layout shown in Figure 1, and suppose that regions $A, B, C$, and $D$ are all to be defined as straight channels. As the 


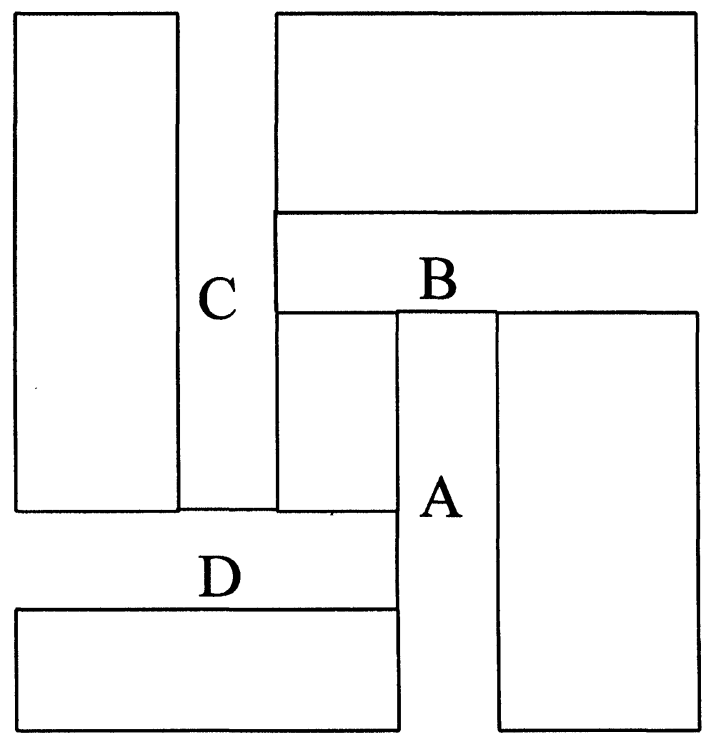

FIGURE 1 A cyclic channel precedence constraint.

terminals in channel $B$ are not fixed, channel $A$ must be routed before channel $B$. Similarly, channels $B, C$, and $D$ must be routed before channels $C$, $D$, and $A$, respectively. Clearly, the iterative precedence relations among channels $A, B, C$ and $D$ form a channel-precedence cycle in this layout. For a building block layout, such a cyclic channel constraint is defined as a cyclic precedence constraint [3].

Basically, if there is any cyclic precedence constraint in a building block layout, routing space will not be fully separated and defined as straight channels to guarantee a safe routing ordering, and such a layout will be defined as a non-slicing layout. For a non-slicing layout, the definition of $L$ shaped channels [3] or switchboxes [4-6] is always introduced to break all the cyclic precedence constraints to guarantee a safe routing ordering in the detailed-routing phrase. If a switchboxbased approach is used to break the cyclic precedence constraint in Figure 1, the height of region $A$ will be estimated according to the routability requirements, and region $A$ will be defined as a switchbox. Furthermore, floating pins on the boundary between regions $A$ and $B$ will be preassigned. Then region $B$, region $C$ and region $D$ will be sequentially defined as straight channels and routed by a straight channel router in that order. Finally, region $A$ will be routed by a switchbox router. However, a successful routing depends on floating pin assignment in one $T$-type junction region, and the performance of a switchbox router. To avoid the layout re-construction and the rip-up and re-route process in the routing phase, floating pin assignment between region $A$ and $B$ is seriously based on a better ordering of the global nets in the $T$-type junction region. For a routing region, the ordering assignment of global nets is called as the crossing distribution problem. It is well known that the crossing distribution problem is also crucial in certain architectures of programmable gate arrays [7] or in the routing of analog and high speed circuits where wires run in parallel for limited distances and additional restrictions on the topology of wires.

For some routing systems [8-9], the crossing distribution problem has been considered as a part of the detailed-routing phase. The PI routing system [10] was the first to introduce an iterative forcedirected "cross-placement" algorithm to solve the crossing distribution problem between any pair of adjacent regions. However, it did not use any channel information to improve the channel density or reduce the routing constraints. In Magic [11], the crossing distribution problem was considered as a part of the global-routing phase. Several algorithms focus on the minimization of wire crossing and twisting conditions. Furthermore, a "buoyancy-calculation" technique [12] was proposed to prevent twisted wires on a region basis, and an improved algorithm [13] later adopted in TimberWolfMC [14] was proposed to minimizes the number of crossings between global nets in the entire layout. Clearly, based on the consideration of via number and total wire length in the routing phase, these approaches only focus on the minimization of net crossings in the crossing distribution problem.

Currently, based on the techniques of topological sorting and perfect matching, M. M. Sadowska and M. Sarrafzadeh also proposed an $O\left(m n^{2}+m c^{3 / 2}\right)$ 
algorithm [15] to distribute all the net crossings into all the regions in a macro cell layout in the quota crossing distribution problem. Basically, the quota in each region was decided by routability consideration and routing constraints in this region. However, the routability consideration and the routing constraints in each region was not further discussed in detail, hence, it is difficult to image how to calculate the quota in one region. As the concept of region definition and ordering assignment is introduced in the design automation of a building block layout, it is clear that a net ordering and net crossings in one $T$-type junction region can be decided by a straight channel router and a switchbox router. For the crossing distribution problem, it is not necessary to simultaneously assign all the net crossings to all the regions in a building block layout. Clearly, it is sufficient to assign a net ordering on the boundary between two adjacent regions according to the routing order in the RDAOA phase. Based on the techniques of computational geometry, D. C. Wang and C. B. Shung [16] proposed an $O(n \log n)$ algorithm for the quota crossing distribution problem, an $O\left(n^{2}\right)$ algorithm for the membership crossing distribution problem and an $O\left(n^{2}\right)$ algorithm for the combination of the quota crossing distribution problem and the membership crossing distribution problem to distribute net crossings into two adjacent regions. However, these algorithms didn't still consider how the quota and membership were obtained from the routability consideration and the routing constraints in one region. To the best of our knowledge, no approach was proposed to combine the routability consideration and the routing constraints into the crossing distribution problem. Since the crossing distribution problem is to distribute all the nets crossings to all the regions to improve routing performance, the crossing distribution problem will consider the routability consideration and the routing constraints instead of the quota and membership values.

Refer to the separation of the routing space in Yan's switchbox-based approach [6], it is clear that only $T$-type junction regions are considered to obtain a net ordering for the crossing distribution problem. In this paper, based on non-crossing relations, vertical constraint relations and netgeometry relations in one $T$-type junction region, two kinds of routability crossing distributions are proposed to improve routing performance in one $T$-type junction region. For routability crossing distribution in one $T$-type junction region, a routability-ordering graph is built to decide a net ordering in $O\left(n^{2}\right)$ time. For routability quota crossing distribution in one $T$-type junction region, if the number of net crossings in base channel is more than a given quota, this net ordering in routability crossing distribution will be further adjusted to satisfy the quota requirement by using a net interchange operation in $O(n)$ time. For floating pin assignment in one $T$-type junction region, global nets are assigned on the boundary between top channel and base channel by interleaving vacant pins in $O(n)$ time according to a net ordering in routability crossing distribution.

\section{PROBLEM DESCRIPTION AND DEFINITIONS}

As mentioned above, for any cyclic precedence constraint in a building block layout, a switchboxbased approach uses the definition of a switchbox to break the cyclic precedence constraint. It is clear that only $T$-type junction regions are considered to obtain a net ordering for the crossing distribution problem. Therefore, it is sufficient for the crossing distribution problem to distribute net crossings on one $T$-type junction region in a building block layout. For one $T$-type junction region $R$, region $R$ is divided into top channel $R_{\text {top }}$ and base channel $R_{\text {base }}$ by one boundary $B$ in the crossing distribution problem. In Figure 2, its division of one $T$ type junction region is shown.

Let us consider that the nets in $N$ are all two-pin nets. For the crossing distribution problem, all the nets in $N$ must intersect boundary $B$, i.e., one pin is located on $R_{\text {top }}$ and the other is located on $R_{\text {base }}$. By dividing the boundary $B$, region $R$ can be 


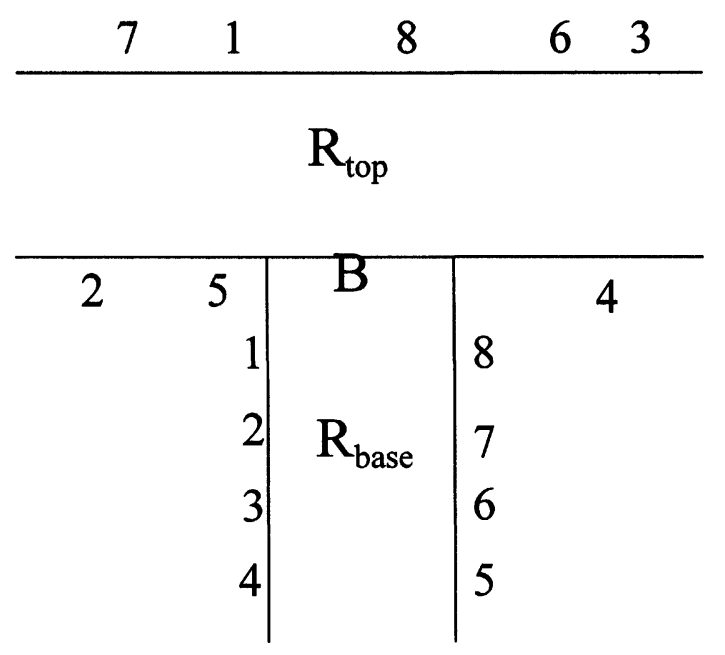

FIGURE $2 T$-type junction region for crossing distribution.

modeled as one straight channel by two pin lists $P_{\text {top }}$ and $P_{\text {base, }}$, where $P_{\text {top }}$ represents the ordering of pins in $R_{\text {top }}$ and $P_{\text {base }}$ represents the ordering of pins in $R_{\text {base }}$ from left to right. In Figure 3, a straight channel modeling the $T$-type junction region in Figure 2 is shown. For net $i$, net $i$ has a top pin in the position of $P_{\text {top }}(i)$ and a base pin in the position of $P_{\text {base }}(i)$, where $P_{\text {top }}(i)$ is the position of net $i$ in $P_{\text {top }}$ and $P_{\text {base }}(i)$ is the position of net $i$ in $P_{\text {base. }}$.

For any pair of nets $i$ and $j$, nets crossings between nets $i$ and $j$ are divided into forced

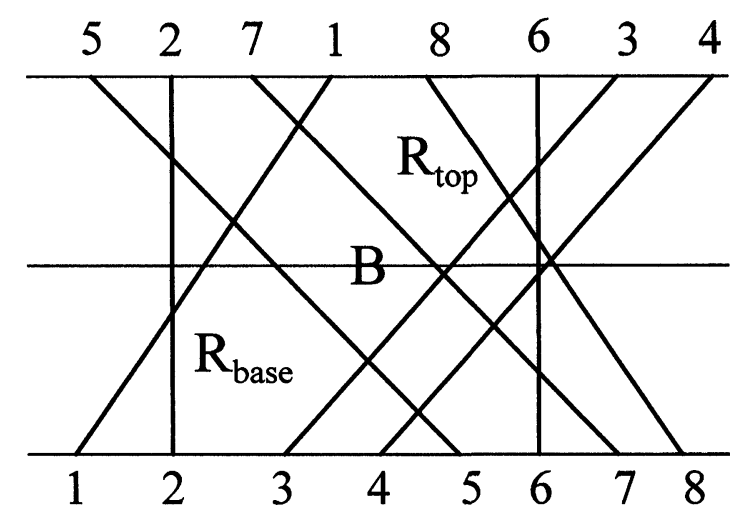

FIGURE 3 Channel modeling for one $T$-type junction region. crossings and redundant crossings. Basically, forced crossing between nets $i$ and $j$ cannot be avoided and redundant crossings can be further deleted in crossing distribution. For any net crossing $(i, j)$, the crossing $(i, j)$ is unordered, i.e., $(i, j)=(j, i)$.

Definition 1 (Forced and Redundant Crossing) For two nets $i$ and $j, i, j \in N$, a forced crossing $(i, j)$ between nets $i$ and $j$ exists if $P_{\text {base }}(i)<P_{\text {base }}(j)$ and $P_{\text {top }}(i)>P_{\text {top }}(j)$ or $P_{\text {base }}(i)>P_{\text {base }}(j)$ and $P_{\text {top }}$ $(i)<P_{\text {top }}(j)$, where $s<t(s>t)$ means that $s$ is located on $t$ 's left (right). On the other hand, if one net crossing $(i, j)$ is not a forced crossing, the crossing $(i, j)$ will be defined as a redundant crossing.

Based on the definition of forced crossings, the set of forced crossings $S_{\text {force }}$ in one $T$-type junction region will be obtained as $\left\{(i, j)\right.$ or $(j, i) \mid P_{\text {base }}(i)<$ $P_{\text {base }}(j)$ and $P_{\text {top }}(i)>P_{\text {top }}(j)$ or $P_{\text {base }}(i)>P_{\text {base }}(j)$ and $P_{\text {top }}(i)<P_{\text {top }}(j)$, for $\left.i, j \in N\right\}$. The $T$-type junction region in Figure 2 has 14 forced crossings, and $S_{\text {force }}=\{(1,2),(1,5),(1,7),(2,5),(3,5),(3,6),(3,7)$, $(3,8),(4,5),(4,6),(4,7),(4,8),(6,7),(6,8)\}$.

Traditionally, the crossing distribution problem [15-16] is to delete all the redundant crossings between two adjacent regions and distributes all the forced crossings into two adjacent regions according to a given quota or membership value. As one crossing distribution region is one $T$-type junction region, the routability and routing constraints will be considered into the crossing distribution problem. Hence, the crossing distribution problem on one $T$-type junction region is called as the routability crossing distribution problem. The routability crossing distribution problem in one $T$-type junction region $R$ is to arrange a net ordering of global nets on $B$ such that no redundant crossing is introduced and forced crossings are distributed in $R$.

It is well known that redundant net crossings yields redundant vias and extra length of wires in the routing phase. Since routability crossing distribution is to guarantee that no redundant crossings are introduced, the non-crossing relations in one $T$-type junction region will be defined to restrict 
the appearance of redundant net crossings in routability crossing distribution.

Definition 2 (Non-Crossing Relations, $N$ ) For pin lists $P_{\text {top }}$ and $P_{\text {base }}$ in one $T$-type junction region, one non-crossing relation $i \rightarrow j$ is defined if $P_{\text {base }}(i)<P_{\text {base }}(j)$, for $i, j \in N$ and $(i, j) \notin S$, and there exists no $k$ such that $P_{\text {base }}(i)<P_{\text {base }}(k)<$ $P_{\text {base }}(j)$, and $P_{\text {top }}(i)<P_{\text {top }}(k)<P_{\text {top }}(j)$.

For the $T$-type junction region in Figure 2 , the set of non-crossing relations is $\{1 \rightarrow 3,1 \rightarrow 6,2 \rightarrow 3$, $2 \rightarrow 7,3 \rightarrow 4,5 \rightarrow 6,5 \rightarrow 7,7 \rightarrow 8\}$.

For the routability consideration and the routing constraints in $R_{\text {top }}$ and $R_{\text {base }}$, the region $R_{\text {top }}$ is further divided into sub-regions $R_{\text {top,left }}, R_{\text {top,middle }}$ and $R_{\text {top,right }}$. In Figure 4 , the $T$-type junction region $R$ is further divided into $R_{\text {top,left }}, R_{\text {top,middle, }}$ $R_{\text {top,right }}$ and $R_{\text {base }}$.

In general, channel density, column densities and the number of vertical constraints in one channel are usually used to estimate the number of tracks in this channel. For one $T$-type junction region $R$, the number of vertical constraints in $R_{\text {base, }}$, the column densities in $R_{\text {top,middle }}$ and the channel density in $R_{\text {top }}$ will be considered in routability crossing distribution. Since one $T$-type junction region $R$ is separated and defined as one

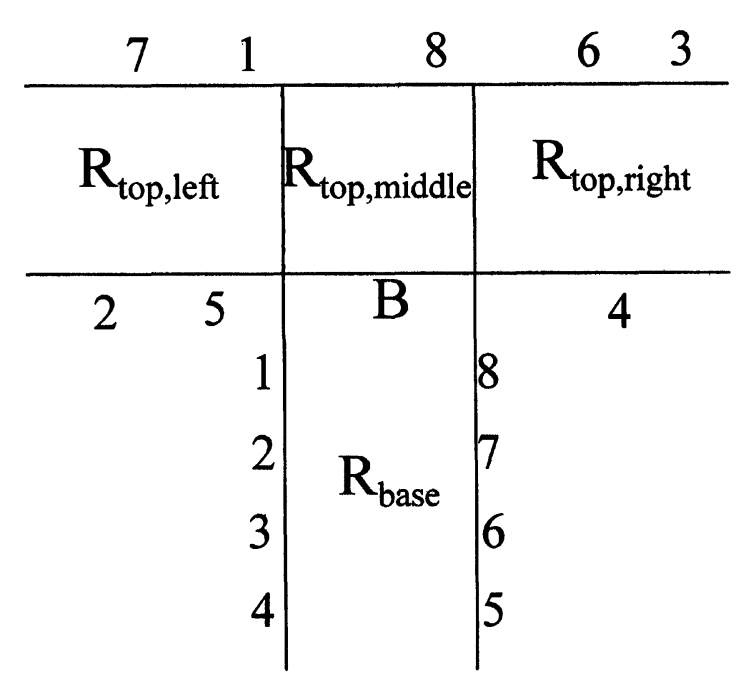

FIGURE 4 Division of one $T$-type junction region. straight channel for $R_{\text {top }}$ and one switchbox for $R_{\text {base }}$ in the RDAOA phase, the vertical constraints in $R_{\text {base }}$ will be avoided to guarantee the routability of region $R_{\text {base }}$ in routability crossing distribution. Hence, the vertical-constraint relations in $R_{\text {base }}$ will be defined to restrict the appearance of vertical constraints in region $R_{\text {base }}$ as follows:

Definition 3 (Vertical-Constraint Relations, $V$ ) For $R_{\text {base }}$ in one $T$-type junction region $R$, one vertical-constraint relation $i \rightarrow j$ in $R_{\text {base }}$ is defined

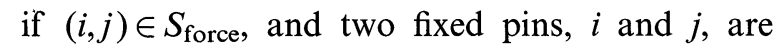
located on the same routing column in $R_{\text {base }}$, where $i$ is located on the left boundary of $R_{\text {base }}$ and $j$ is located on the right boundary of $R_{\text {base }}$.

For the $T$-type junction region in Figure 2, the set of vertical-constraint relations is $\{3 \rightarrow 6$, $4 \rightarrow 5\}$.

Furthermore, to reduce the column densities in $R_{\text {top,middle }}$ or the channel density in $R_{\text {top }}$, the netgeometry relations among $R_{\text {top,left }}, R_{\text {top,middle }}$ and $R_{\text {top,right }}$ will be further defined to reduce the number of tracks, total wire length and the number of vias in the channel $R_{\text {top. }}$.

Definition 4 (Net-Geometry Relations, $G$ ) For regions $R_{\text {top,left }}, R_{\text {top,middle }}$ and $R_{\text {top,right }}$, one netgeometry relation $i \rightarrow j$ among $R_{\text {top,left }}, R_{\text {top,middle }}$ and $R_{\text {top,right }}$ is defined if $(i, j) \in S_{\text {force }}$ and nets $i$ and $j$ correspond to one of the three conditions:

Condition 1 one pin of net $i$ is located in $R_{\text {top,left }}$ and one pin of net $j$ is located in $R_{\mathrm{top}, \text { middle, }}$ or $R_{\mathrm{top}, \text { right}}$;

Condition 2 one pin of nets $i$ is located in $R_{\text {top,middle, one pin of nets } i \text { is }}$ located in $R_{\text {top,middle, and } P_{\text {top }}(i)<}$ $P_{\text {top }}(j)$

Condition 3 one pin of net $i$ is located in $R_{\text {top,middle, and one pin of net } j \text { is }}$ located in $R_{\text {top,right; }}$

For the $T$-type junction region in Figure 2, the set of net-geometry relations is $\{7 \rightarrow 6,7 \rightarrow 3,7 \rightarrow 4$, $5 \rightarrow 3,5 \rightarrow 4,8 \rightarrow 6,8 \rightarrow 3,8 \rightarrow 4\}$. 
For one $T$-type junction region $R$, all the noncrossing relations guarantee that no redundant net crossing is introduced in $R$ and all the verticalconstraint relations improve routability in region $R_{\text {base }}$. Hence, all the non-crossing relations and all the vertical-constraint relations in $R$ are fully applied to routability crossing distribution. However, it is possible that one net-geometry relation in $R_{\text {top }}$ will conflict with one vertical-constraint relation in $R_{\text {base }}$. Since the routability in $R_{\text {base }}$ is more important that in $R_{\text {top }}$, the net-geometry relations may not be fully applied to routability crossing distribution. Therefore, based on the application of non-crossing relations, verticalconstraint relations and net-geometry relation in one $T$-type junction region, the routability crossing distribution problem is modeled as follows:

\subsection{Routability Crossing Distribution}

Given one $T$-type junction region $R$ and a set of two-pin global nets, the routability crossing distribution problem is to find a feasible ordering of global nets such that the net ordering satisfies all of the non-crossing relations, all of the verticalconstraint relations and a maximal set of netgeometry relations in $R$.

As mentioned above, one $T$-type junction region is divided into a straight channel and a switchbox by assigning floating pins on the boundary in the RDAOA phase. It is well known that the channel height is adjustable and the switchbox height is not adjustable in the routing phase. Hence, the routability of a switchbox is more important than that of a channel in the routing phase. To route successfully one $T$-type junction region, a small quota $K$ (even to $\mathbf{0}$ ) is selected to guarantee that the divided switchbox can be successfully routed. Thus, the routability quota crossing distribution problem will be modeled as follows:

\subsection{Routability Quota Crossing Distribution}

Given one $T$-type junction region $R$, a set of twopin global nets and an integer quota $K$, the routability quota crossing distribution problem is to find a feasible ordering of global nets such that the net ordering makes at most $K$ net crossings in $R_{\text {base, }}$, and satisfies all of the non-crossing relations, all of the vertical-constraint relations and a maximal set of net-geometry relations in $R$.

According to a net ordering obtained in routability crossing distribution, floating pins on the boundary $B$ are further assigned to minimize the number of vertical constraints in $R_{\text {top,middle. }}$. Hence, the floating pin assignment problem will be modeled as follows:

\subsection{Floating Pin Assignment}

Given one $T$-type junction region $R$, a feasible net ordering, and a vertical constraint graph in $R_{\text {top }}$, the floating pin assignment problem is to assign all of the global nets on B such that no constraint cycle is yielded and the number of vertical constraints in $R_{\text {top,middle }}$ is minimized.

\section{ROUTABILITY CROSSING DISTRIBUTION}

For routability crossing distribution in one $T$-type junction region $R$, a net ordering of global nets is obtained by using non-crossing relations, verticalconstraint relations and net-geometry relations in $R$. In this section, two kinds of routability crossing distributions are considered to decide a net ordering of global nets on the boundary between $R_{\text {top }}$ and $R_{\text {base. }}$ One is a pure routability crossing distribution. Based on the definitions of noncrossing relations, vertical-constraint relations and net-geometry relations in $R$, a net ordering of global nets is obtained by running a modified topological sorting on a routability-ordering graph. The other is a routability quota crossing distribution. To guarantee $R_{\text {base }}$ to be successfully routed, one quota $K$ in $R_{\text {base }}$ is estimated according to routability constraints in $R_{\text {base }}$. Based on a net ordering in routability crossing distribution, if the number of net crossings in $R_{\text {base }}$ is more than 
$K$, the net ordering will be modified to satisfy the quota restriction by using a net interchange operation.

\subsection{Net Ordering for Routability Crossing Distribution}

According to the definition of non-crossing relations in $R$, a directed non-crossing ordering graph $G=(V, A)$ is built, where $v$ in $V$ represents a global net and $e$ in $A$ represents a non-crossing relation. Consider the $T$-type junction region in Figure 2, the set of non-crossing relations is $\{1 \rightarrow 3,1 \rightarrow 6$, $2 \rightarrow 3,2 \rightarrow 7,3 \rightarrow 4,5 \rightarrow 6,5 \rightarrow 7,7 \rightarrow 8\}$ and its non-crossing ordering graph is shown in Figure 5.

THEOREM 1 Any non-crossing ordering graph $G$ is acyclic.

Since any non-crossing ordering graph is acyclic, a net ordering for a non-crossing ordering graph will be easily obtained by using a topological sorting. By the restriction of a non-crossing relation in $R$, any pair of global nets with a non-

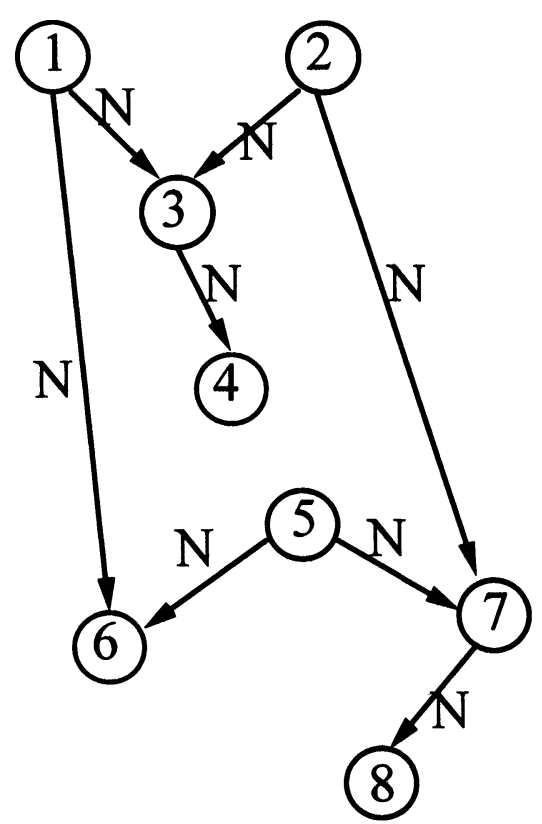

FIGURE 5 A non-crossing ordering graph for the $T$-type junction region in Figure 2. crossing relation is certainly no net crossing in this net ordering. Hence, the number of net crossings is minimized and no redundant net crossing is yielded in this net ordering.

THEOREM 2 A net ordering generated from a noncrossing ordering graph will yield no redundant net crossing.

According to the definitions of non-crossing relations and vertical-constraint relations in $R$, a directed constraint-ordering graph $G^{*}=\left(V^{*}, A^{*}\right)$ is built, where $v$ in $V^{*}$ represents a global net and $e$ in $A^{*}$ represents a non-crossing relation or a vertical-constraint relation. Consider the $T$-type junction region in Figure 2, the set of vertical-constraint relations is $\{3 \rightarrow 6,4 \rightarrow 5\}$ and its constraint-ordering graph is shown in Figure 6.

THEOREM 3 Any constraint-ordering graph $G^{*}$ is acyclic.

Since any constraint-ordering graph is acyclic, a net ordering for a constraint-ordering graph will be obtained by using a topological sorting. Based

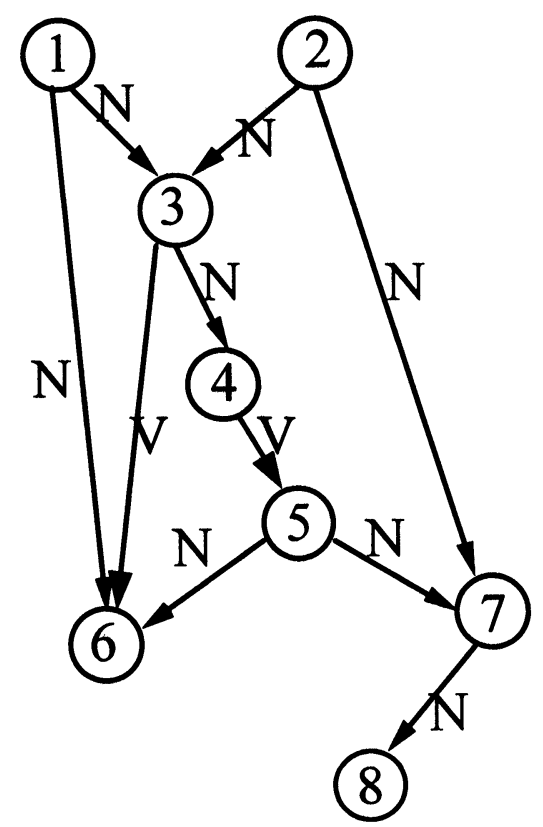

FIGURE 6 A constraint-ordering graph for the $T$-type junction region in Figure 2. 
on the restriction of a vertical-constraint relation in $R_{\text {base }}$, if there is a vertical constraint relation between one pair of global nets with a forced crossing, this forced crossing will be pushed into $R_{\text {top. }}$. Hence, a net ordering which generated from a constraint-ordering graph will guarantee that no forced crossing is distribution in $R_{\text {base }}$.

THEOREM 4 A net ordering generated for a constraint ordering graph will yield no redundant net crossings and guarantee that no forced crossing is distribution in $R_{\text {base }}$.

According to the definitions of non-crossing relations, vertical-constraint relations, and netgeometry relations in $R$, a directed routabilityordering graph $G^{* *}=\left(V^{* *}, A^{* *}\right)$ is built, where $\mathrm{v}$ in $V^{* *}$ represents a global net and $e$ in $A^{* *}$ represents a non-crossing relation, a vertical-constraint relation or a net-geometry relation. Consider the $T$ type junction region in Figure 2, the set of netgeometry relations is $\{7 \rightarrow 6,7 \rightarrow 3,7 \rightarrow 4,5 \rightarrow 3$, $5 \rightarrow 4,8 \rightarrow 6,8 \rightarrow 3,8 \rightarrow 4\}$ and its routability-ordering graph is shown in Figure 7.

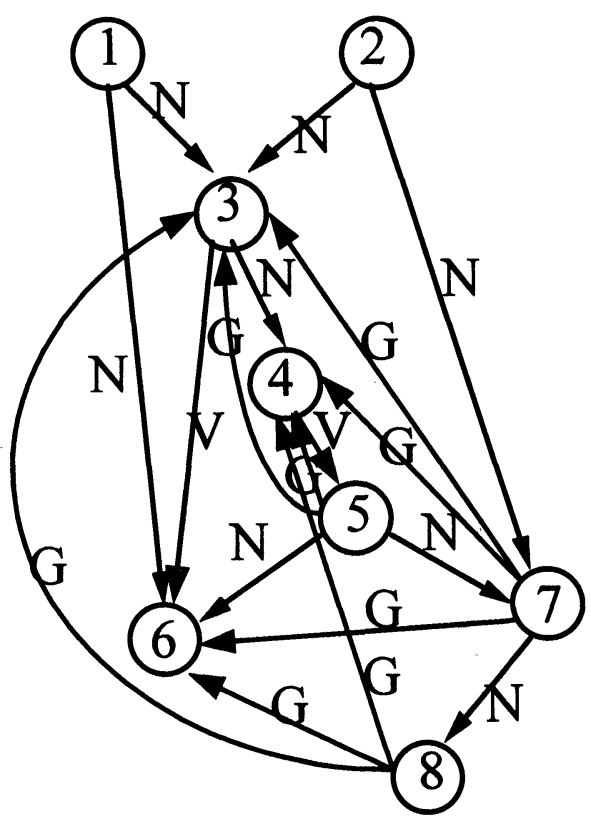

FIGURE 7 A routability-ordering graph for the $T$-type junction region in Figure 2.
For one $T$-type junction region $R$, it is possible that a net-geometry relation in $R_{\text {top }}$ conflicts with a vertical-constraint relation in $R_{\text {base }}$. Hence, as all the net-geometry relations in $R_{\text {top }}$ are introduced into a constraint-ordering graph, the resultant routability-ordering graph may be cyclic. If a routability-ordering graph is cyclic, all the noncrossing relations, all the vertical-constraint relations and a maximal set of net-geometry relations in $R$ will be applied to improve the routing performance in routability crossing distribution.

By using a modified topological sorting, a net ordering of global nets is obtained to satisfy all the non-crossing relations, all the vertical-constraint relations and a maximal set of net-geometry relations in $R$. In this modified topological sorting, any vertex without any predecessor is ordered and then deleted from this graph if a routabilityordering graph is not empty. On the other hand, if there exists no vertex without any predecessor in this graph, one vertex with only net-geometry predecessors will be ordered and then deleted from this graph. Finally, a routability net ordering is obtained. It is well known that if the data structure of a graph $G=(V, A)$ is an adjacency list, the time complexity of a topological sorting is in $O(|V|+|A|)$ time. Since $O\left(\left|A^{* *}\right|\right)=O\left(n^{2}\right)$ in the worst case, the time complexity in routability crossing distribution is in $O\left(n^{2}\right)$ time, where $n$ is the number of global nets in $R$. The topological sorting algorithm, Routability_Crossing_Distribution, for routability crossing distribution is described as follows:

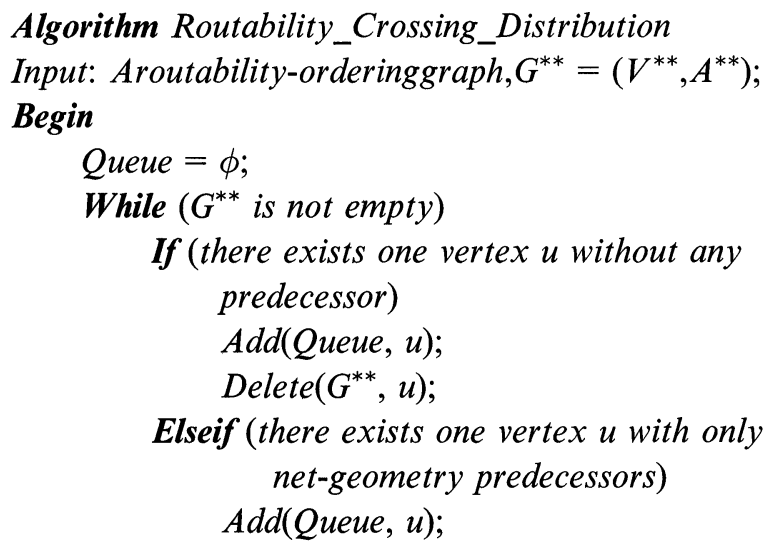

Algorithm Routability_Crossing_Distribution Input: Aroutability-orderinggraph, $G^{* *}=\left(V^{* *}, A^{* *}\right)$; Begin 


\section{$\operatorname{Delete}\left(G^{* *}, u\right)$ \\ Endif \\ Return(Queue); \\ End}

By using this algorithm, Routability_Crossing_ Distribution, a net ordering in the $T$-type junction region in Figure 2 is obtained as $(1,2,3,4,5,7,8$, 6) or $(2,1,3,4,5,7,8,6)$.

\subsection{Net Ordering for Routability Quota Crossing Distribution}

By using this algorithm, Routability_Crossing_Distribution, a net ordering of global nets is obtained for routability crossing distribution. Given a routing quota $K$ in $R_{\text {base, }}$, if the number of crossings in $R_{\text {base }}$ is more than $K$, the net ordering will be modified to satisfy the quota restriction in routability quota crossing distribution by using a net interchange operation. Consider that a net ordering $\left(\mathrm{Net}_{1}, \mathrm{Net}_{2}, \ldots, \mathrm{Net}_{n}\right)$ in routability crossing distribution, if $P_{\text {base }}\left(\mathrm{Net}_{i}\right)>P_{\text {base }}\left(\mathrm{Net}_{i+1}\right)$ in $\left(\mathrm{Net}_{1}, \mathrm{Net}_{2}, \ldots, \mathrm{Net}_{n}\right)$, for $1 \leq i \leq n-1$, there will exist one forced crossing in $R_{\text {base. }}$ If the orders of $\mathrm{Net}_{i}$ and $\mathrm{Net}_{i+1}$ are interchanged, the crossing between $\mathrm{Net}_{i}$ and $\mathrm{Net}_{i+1}$ will be pushed into $R_{\text {top }}$. To further satisfy the quota $K$, net interchanges will be done to reduce the number of crossings in $R_{\text {base }}$ for routability quota crossing distribution. As a result, a modified net ordering for routability quota crossing distribution will be obtained by using a net interchange operation. The net-interchange algorithm, Routability_Quota_Crossing_ Distribution, will be described as follows:

\section{Algorithm Routability_Quota_Crossing_Distribu- tion}

Input: $A$ net ordering $\left(\mathrm{Net}_{1}, \mathrm{Net}_{2}, \ldots, \mathrm{Net}_{n}\right)$ and $a$ routing quota $K$;

\section{Begin}

Crossing_Number $=$ the number of net crossings in $R_{\text {base }}$ according to the net ordering $\left(\mathrm{Net}_{1}, \mathrm{Net}_{2}, \ldots, \mathrm{Net}_{n}\right)$;

While (Crossing_Number $>K$ )
Begin

Find a pair of Net $t_{i}$ and $\mathrm{Net}_{i+1}$ such that $P_{\text {base }}\left(N e t_{i}\right)>P_{\text {base }}\left(N e t_{i+1}\right)$;

Modify the net ordering ( $\mathrm{Net}_{1}$, $\left.\mathrm{Net}_{2}, \ldots, \mathrm{Net}_{i}, \mathrm{Net}_{\mathrm{i+1}}, \ldots, \mathrm{Net}_{n}\right)$ into $\left(\mathrm{Net}_{1}, \mathrm{Net}_{2}, \ldots, \mathrm{Net}_{i+1}, \mathrm{Net}_{i}, \ldots, \mathrm{Net}_{n}\right)$; Crossing_Number $=$ Crossing_Number -1 ; End

Return a net ordering such that the number of net crossings in $R_{\text {base }}$ is at most $K$;

\section{End}

Since $K$ is known and a net ordering of global nets is decided in routability crossing distribution, the number of crossings in $R_{\text {base }}$ can be easily calculated. In this algorithm, Routability_Quota_ Crossing_Distribution, the iterative number in while-loop statement is $O(1)$, and the time complexity of finding a pair of nets $\mathrm{Net}_{i}$ and $\mathrm{Net}_{i+1}$ is in $O(n)$ time. Hence, the time complexity of this algorithm is in $O(n)$ time. Clearly, a modified net ordering in routability quota crossing distribution is obtained in $O\left(n^{2}\right)$ time.

For the $T$-type junction region in Figure 2, if a net ordering is $(1,2,3,4,5,7,8,6)$ or $(2,1,3,4,5$, $7,8,6)$, the number of nets crossing in $R_{\text {base }}$ is 2 or 3 , respectively. If $K$ is 1 , two net orderings will be modified into $(1,2,3,4,5,7,6,8)$ at the same time to satisfy the quota constraint in routability quota crossing distribution.

\subsection{Extension to Multiple-pin Nets}

For routability crossing distribution, all the routing nets in one $T$-type junction region are considered as two-pin nets. However, it is impossible that all the routing nets in one $T$-type junction region are twopin nets. Hence, multiple-pin nets are further considered in one $T$-type junction region for routability crossing distribution.

Basically, a global router assigns the wiring paths of all the global nets on the routing space. To satisfy the routing requirements, the wiring path of any multiple-pin net only crosses the boundary in one- $T$-type junction region at most 


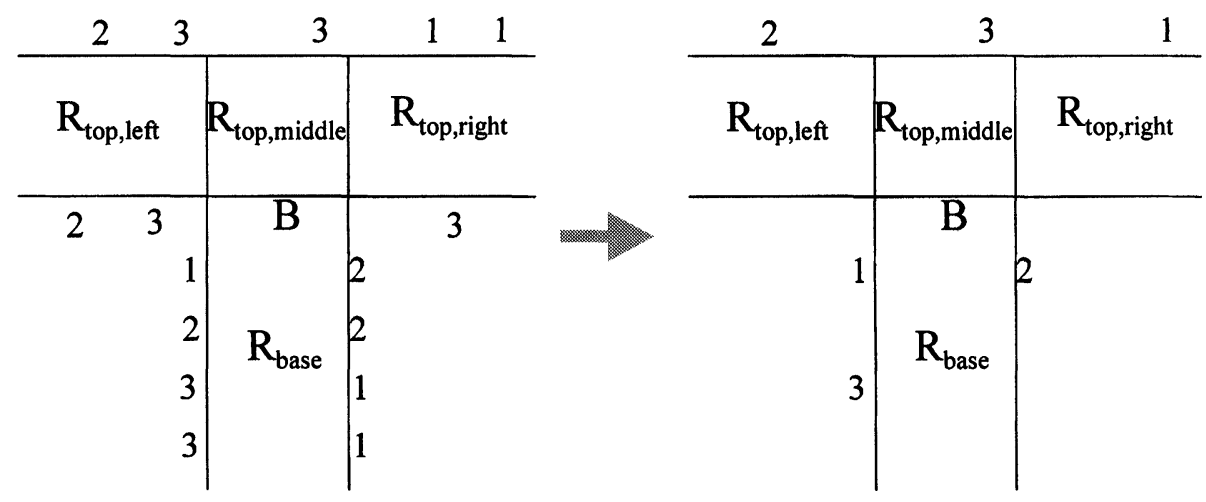

FIGURE 8 Pin selection of multiple-pin nets.

one time. If the wiring path of one multiple-pin net crosses the boundary $B$ in $R$, some pins in this net will be located in $R_{\text {top }}$ and the others will be located in $R_{\text {base }}$. Due to the consideration of routing performance in the global-routing phase, it is clear that there exists only one crossing between any multiple-pin net and the boundary $B$ in $R$. Due to the doglegging introduction in the detailedrouting phase, only one segment in any multiplepin net which intersects the boundary $B$ is considered for routability crossing distribution. For any multiple-pin net, one representative pin in $R_{\text {top }}$ and the other representative pin in $R_{\text {base }}$ are selected to construct one intersecting segment for routability crossing distribution.

For any multiple-pin net, one pin in $R_{\text {base }}$ that is the most near to the boundary $B$ is selected as the representative in $R_{\text {base. }}$ On the other hand, the selection of one representative pin in $R_{\text {top }}$ will be considered in the following three cases:

Case 1 If all the pins in $R_{\text {top }}$ are located in $R_{\text {top, left }}$, and $R_{\text {top,right }}$, one vacant pin in $R_{\text {top,middle }}$ is selected as the representative in $R_{\text {top }}$ if there is one vacant pin in $R_{\text {top,middle }}$.

Case 2 If all the pins in $R_{\text {top }}$ are located in $R_{\text {top,left }}$ or $R_{\text {top,right, }}$ one pin in $R_{\text {top,left }}$ or $R_{\text {top,right }}$ that is the most near to the boundary $B$ is selected as the representative in $R_{\text {top. }}$.

Case 3 If not, one pin in $R_{\text {top,middle }}$ is selected as the representative in $R_{\text {top. }}$.
Since all the multiple-pin nets in one $T$-type junction region use two-pin intersecting segments for routability crossing distribution, the process of finding a feasible net ordering of two-pin intersecting segments is the same as that of two-pin nets for routability crossing distribution. Therefore, it is easy that the proposed approach for routability crossing distribution of two-pin nets can extend for routability crossing distribution of multiple-pin nets. In Figure 8, three multiple-pin nets in one $T$ type junction region use three two-pin intersecting segments for routability crossing distribution.

\section{FLOATING PIN ASSIGNMENT}

Since a net ordering of global nets is obtained for routability crossing distribution or routability quota crossing distribution, these global nets can be assigned onto the boundary $B$ for floating pin assignment. In floating pin assignment, there are fixed net pins and vacant pins on top boundary of $R_{\text {top,middle, and these global nets and vacant pins }}$ are assigned on bottom boundary of $R_{\text {top,middle. If }}$ the number of global nets equals to that of floating pins on $B$, these global nets will be assigned in this order. If the number of global nets is less than that of floating pins on $B$, these global nets will be assigned to minimize the number of vertical constraints in $R_{\text {top,middle }}$ and not to yield any verticalconstraint cycle by adding vacant pins between any pair of adjacent global nets. 

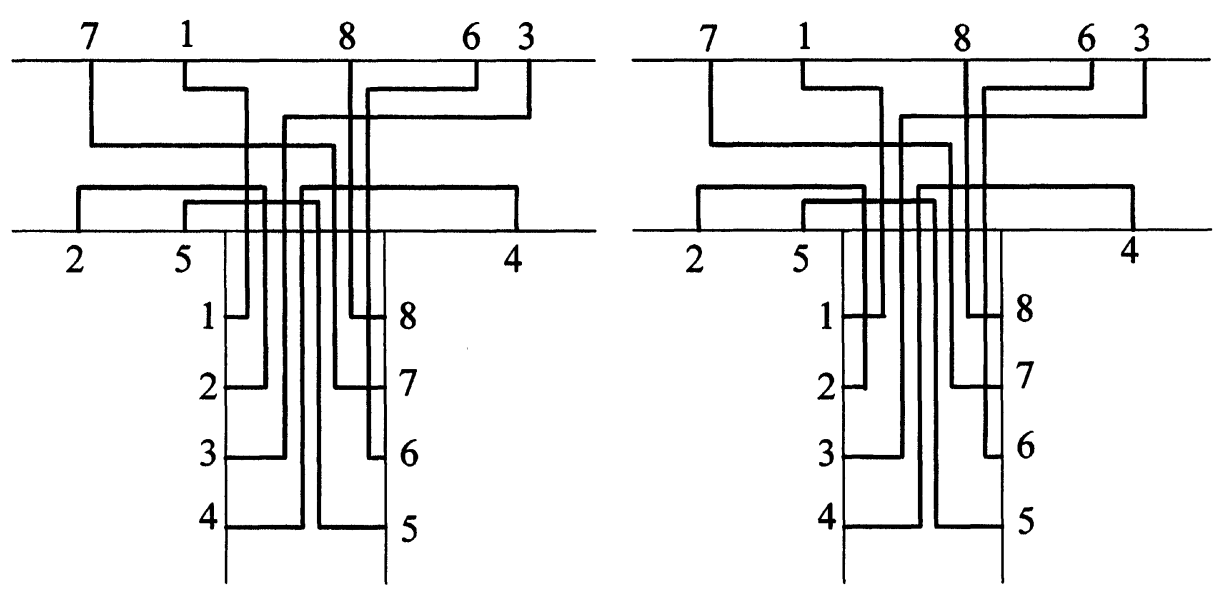

FIGURE 9 Routing results for routability crossing distribution.

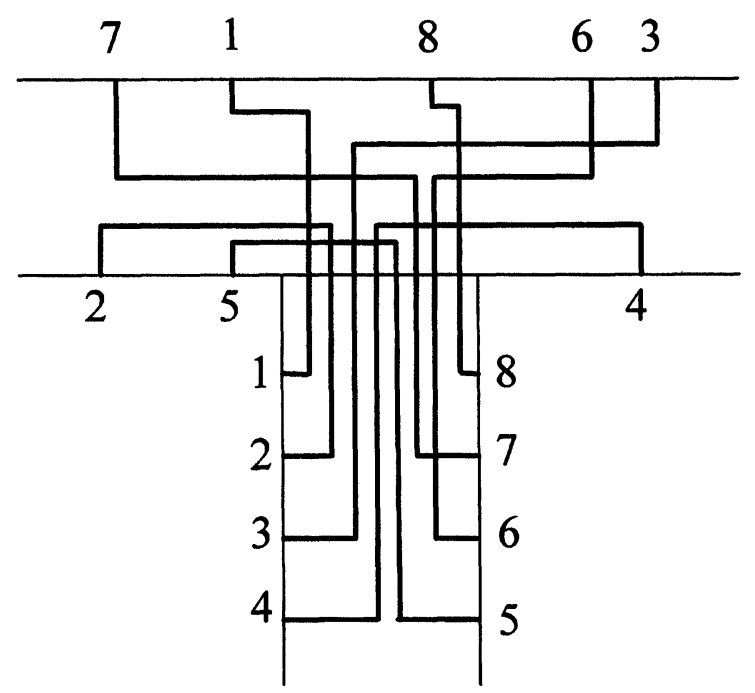

FIGURE 10 Routing result for routability quota crossing distribution with $K=1$.

Let $N_{\text {top,net }}\left(N_{\text {bottom,net }}\right)$ and $N_{\text {top,vacant }}$ ( $N_{\text {bottom,vacant }}$ be the number of net pins and vacant pin on top (bottom) boundary in $R_{\text {top,middle, }}$ respectively. Let $N_{b}$ be the number of floating pins on the boundary $B$. Clearly, $N_{b}=N_{\text {top,net }}+$ $N_{\text {top,vacant }}=N_{\text {bottom,net }}+N_{\text {bottom,vacant }}$. If $N_{\text {top,net }}+$ $N_{\text {bottom,net }} \leq N_{b}$, these global nets in a net ordering will be assigned by interleaving vacant pins on $B$ such that no vertical constraint is introduced in $R_{\text {top,middle }}$ according to the distribution of vacant pins on top boundary of $R_{\text {top,middle }}$. If $N_{\text {top,net }}+$
$N_{\text {bottom,net }}>N_{b}$, there will be at most $\left|N_{\text {top,net }}-N_{\text {bottom,net }}\right|$ vertical constraints in $R_{\text {top,middle }}$ and these vertical constraints will not yield any vertical-constraint cycle in $R_{\text {top }}$. The algorithm, Floating_Pin_Assignment, assigns floating pins on $B$ and is described as follows:

Algorithm Floating_Pin_Assignment Input: $A$ vertical constraint graph in $R_{\text {top }}$, a top pin list $\left(P_{1}, P_{2}, \ldots, P_{N_{b}}\right)$, and a net ordering (Net 1 , $\left.\mathrm{Net}_{2}, \ldots, \mathrm{Net}_{\mathrm{N}_{\text {bottom,net }}}\right)$ 


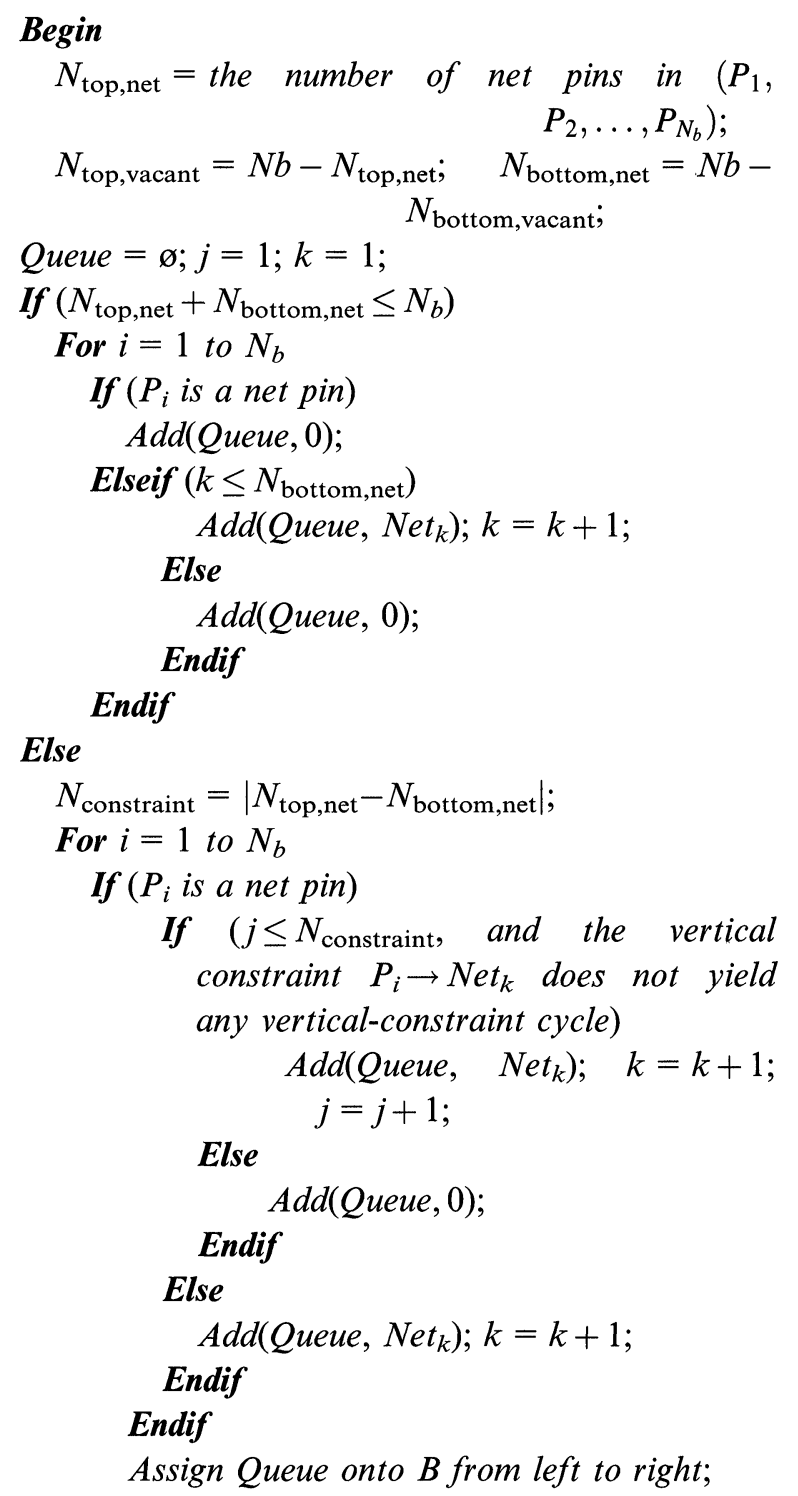

End

Since $N_{b}$ and $\left(P_{1}, P_{2}, \ldots, P_{N_{b}}\right)$ are given, the number of net pins in top boundary of $R_{\text {top, middle }}$ is easily calculated. In the algorithm, Floating_Pin_ Assignment, these global nets in a net ordering are assigned on $B$ by using a sequential checking operation. Hence, the time complexity of this algorithm is in $O(N b+n)$ time, where $n$ is the number of global nets. Since $N_{b}$ is known, the time complexity of this algorithm, Floating_Pin_Assignment, is in $O(n)$ time. According to the results of routability crossing distribution, routability crossing quota distribution with $K=1$ and floating pin assignment in one $T$-type junction region of Figure 2 , the routing results are shown in Figure 9 and Figure 10, respectively.

\section{CONCLUSIONS}

Based on non-crossing relations, vertical constraint relations and net-geometry relations in one $T$-type junction region, two kinds of routability crossing distributions are proposed to improve routing performance in one $T$-type junction region. For routability crossing distribution in one $T$-type junction region, a routabilityordering graph is built to decide a net ordering in $O\left(n^{2}\right)$ time. For routability quota crossing distribution in one $T$-type junction region, if the number of net crossings in base channel is more than a given quota, this net ordering in routability crossing distribution will be further adjusted to satisfy the quota requirement by using a net interchange operation in $O(n)$ time. For floating pin assignment in one $T$-type junction region, global nets are assigned on the boundary between top channel and base channel by interleaving vacant pins in $O(n)$ time according to a net ordering in routability crossing distribution.

\section{References}

[1] Chen, N. P., Hsu, C. P. and Kuh, E. S. (1983). "BBL: A Building Block Layout Systems for Custom Chip Design", International Conference on Computer-Aided Design, pp. $40-41$.

[2] Ohtsuki, T. (1986). Editor, Layout Design and Verification, Amsterdam: North Holland.

[3] Dai, W. M., Asano, T. and Kuh, E. S. (1985). "Routing Region Definition and Ordering Scheme for Building-Block Layout", IEEE Trans. on Computer-Aided Design, 4, $189-197$.

[4] Kajitani, Y. (1983). "Order of Channels for Safe Routing and Optimal Compaction of Routing Area", IEEE Trans. on Computer-Aided Design, 2, 293-300.

[5] Cai, Y. and Wong, D. F. (1991). "Channel/Switchbox Definition for VLSI Building-Block Layout", IEEE Trans. on Computer-Aided Design, 10, 1485-1493.

[6] Yan, J. T. and Hsiao, P. Y. (1996). "Minimizing the Number of Switchboxes for Region Definition and 
Ordering Assignment", IEEE Trans. on Computer-Aided Design of Integrated Circuits and Systems, 15(3), 336-347.

[7] Hsieh, H. C. et al. (1990). "Third-Generation Architecture Boosts Speed and Density of Field-Programmable Gate Array", Custom Integrated Circuits Conference, pp. $31.2 .1-31.2 .7$

[8] Dai, W., Chen, H. H., Dutta, R., Jackson, M., Kuh, E. S., Sadowska, M. M., Sato, M., Wang, D. and Xiong, X. (1987). "Bear: A New Building-Block Layout System", International Conference on Computer-Aided Design, pp. $34-37$.

[9] Groeneveld, P. and Cai, H. (1988). "The Delft Placement and Routing System", International Workshop on Placement and Routing, p. 6.2 .

[10] Rivest, R. L. (1982). "The PI (Placement and Routing) System", 19th Design Automation Conference, pp. 475481.

[11] Hamachi, G. T. and Ousterhout, J. K. (1985). "Magic's Obstacle-Avoiding Global Router", Chappel Hill Conference on Very Large Scale Integration, pp. 145-164.

[12] Ng, C. H. (1989). "A Symbolic-Interconnect Router for Custom IC Design", 26th Design Automation Conference, pp. $52-57$.

[13] Groenveld, P. (1989). "On Global Wire Ordering for Macro-Cell Routing", 26th Design Automation Conference, pp. 155-160.

[14] Schwartz, W. and Sechen, C. (1990). "New Algorithms for the Place and Route of Macro Cells", International Workshop on Placement and Routing, p. 1.2.
[15] Sadowska, M. M. and Sarrafzadeh, M. (1991). "The Crossing Distribution Problem", International Conference on Computer-Aided Design, pp. 528-531.

[16] Wang, D. C. and Shung, C. B. (1992). "Crossing Distribution", the European Design Automation Conference, pp. 354-361.

\section{Author's Biography}

Jin-Tai Yan received the B.S., M.S., and Ph.D. degrees in computer and information science from National Chiao-Tung University, Hsinchu, Taiwan, R.O.C. in 1988, 1990, and 1995, respectively.

From 1995 to 1997 he served in the Chinese Navy, Kaohsiung, Taiwan, as an information officer. Since 1997 he has been with National Chiao-Tung University, Hsinchu, Taiwan, R.O.C. and is currently a post-doctor researcher in Computer Systems Research Center. His current research interests are high-level synthesis, logic synthesis, and physical design of VLSI circuits, parallel/distributed computing, and network performance analysis. 

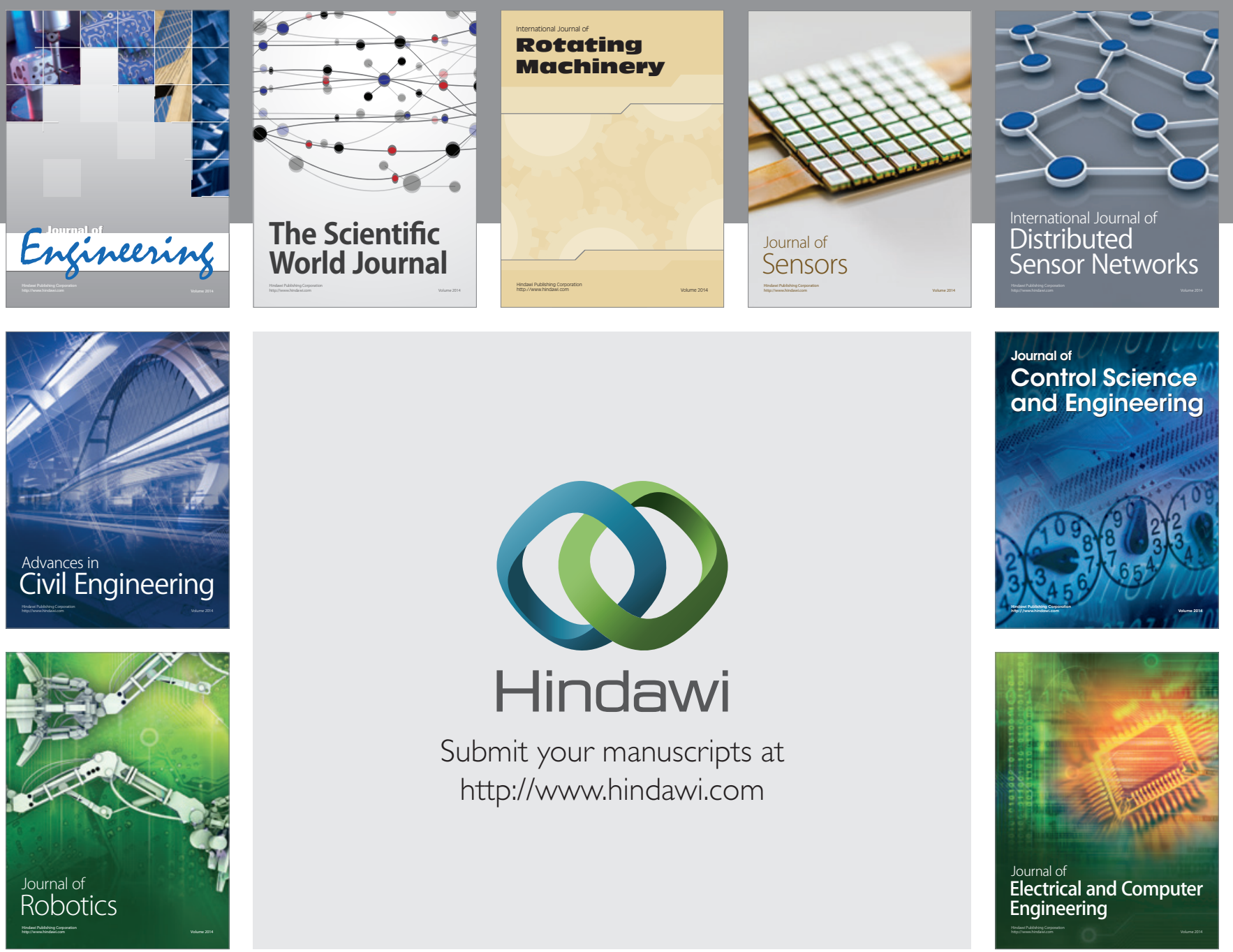

Submit your manuscripts at

http://www.hindawi.com
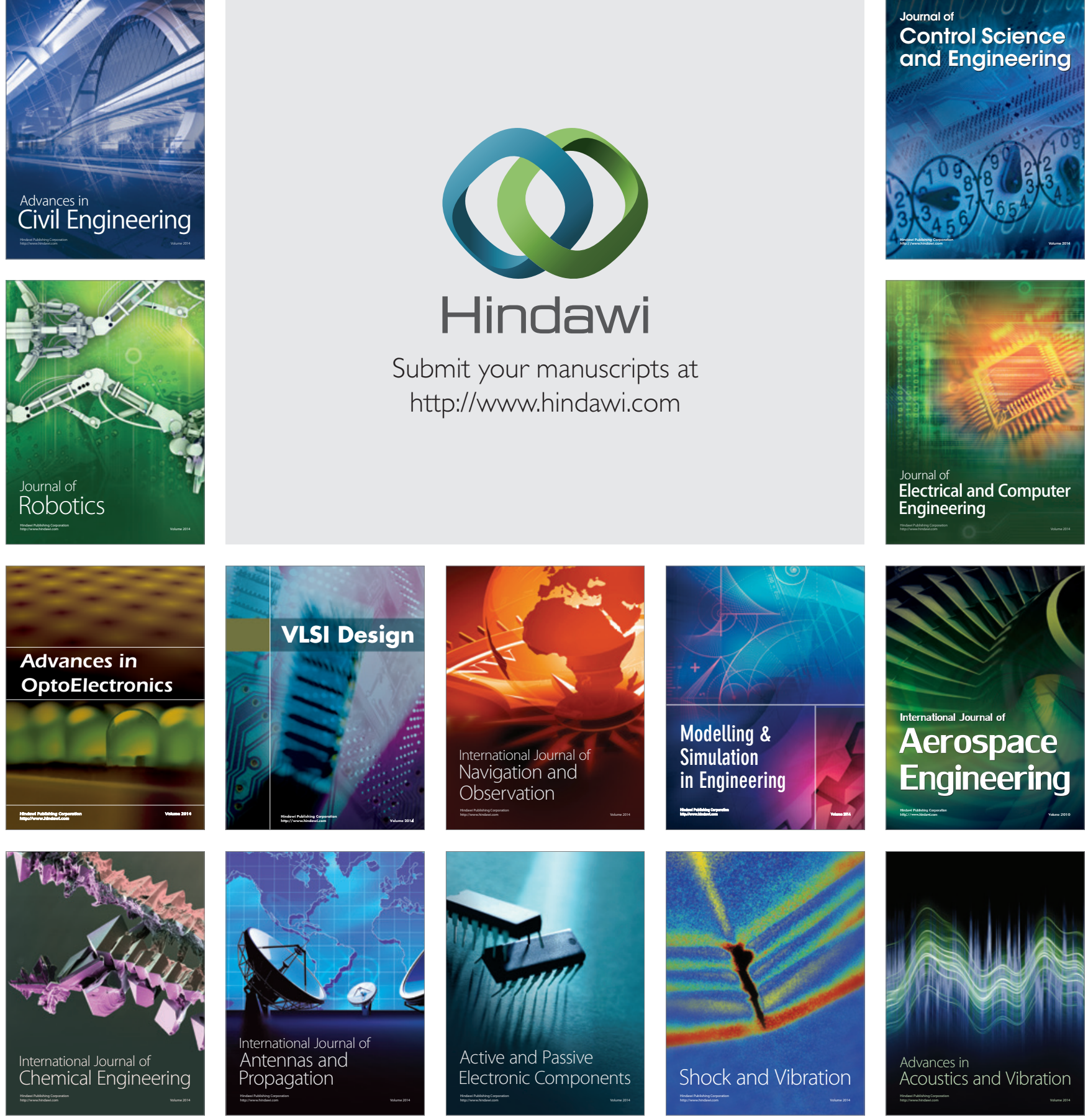